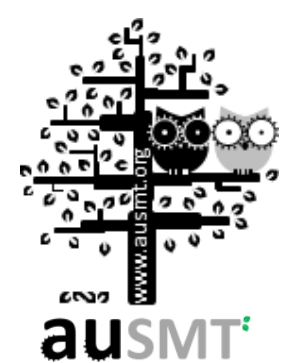

\title{
Development of Tuning Fork-shaped Clamps with Nickel-electroded Ionic Polymer Metal Composites
}

\author{
Guo-Hua Feng* and Wei-Lun Huang \\ University, Taiwan \\ (Received 9 November 2011; Accepted 20 December 2011; Published on line 1 March 2012) \\ *Corresponding author: imeghf@ccu.edu.tw \\ DOI: $10.5875 / a u s m t . v 2 i 1.118$
}

Department of Mechanical Engineering and Advanced Institute of Manufacturing with High-tech Innovations, National Chung Cheng

\begin{abstract}
This study presents an innovative soft tuning fork-shaped clamp transducer consisting of a clamp with two fingers and a handle. We successfully fabricated the clamp, made of ionic polymer metal composites (IPMCs) with nickel electrodes, using a cost-effective micromachining fabrication technique. Further, we conducted experiments to characterize the electrical and mechanical properties of the IPMC clamp. The clamp can be electrically controlled in an open or closed state. Between the two front fingers, a largest gap of $2.5 \mathrm{~mm}$ and a smallest gap of $0.5 \mathrm{~mm}$ were achieved with an $8 \mathrm{~V}$ square wave actuation. The clamp was also demonstrated to continuously pinch an object for 1 min, and the instance in which a sudden external force was exerted on the pinched object could be detected from its feedback current signal.
\end{abstract}

Keywords: IPMC clamp; nickel; biomimetic; micromachining

\section{Introduction}

Ionic polymer metal composites (IPMCs) have been widely studied for use in biomimic transducers due to their promising biomechanical and biomimetic applications [1-3]. IPMC materials demonstrate considerable potential as soft robotic actuators, artificial muscles and dynamic sensors due to their large dynamic deformation under time-varying electric fields [4-6].

The IPMC transducer is typically constructed as a planar structure with Nafion (a copolymer of polytetrafluorethylene (PTFE) and perfluorinated vinyl ether sulfonate) sandwiched between two plated metal electrodes. The hydrophobic PTFE provides a three-dimensional backbone structure, while the individual side chains orient their hydrophilic-end groups to shape as clusters, which are easily saturated with water allowing the cations to move through these interstitial regions under an external electrical field. During actuation, these cations along with water molecules can move toward the anode of an IPMC transducer to generate a bending motion $[6,7]$.

Conventionally, IPMC preparation includes the ionic polymer formation, initial compositing process, and surface electroding process [8-10]. After these processes are completed, the individual IPMC devices are cut according to designed shapes from the large sheet of the sandwiched metal/ionomer/metal structure [11, 12]. As such, relatively large-scale and simple-shaped planar-structured IPMC actuators are commonly investigated.

Most reported IPMC devices focused on a cantilever structure because of the relatively easy fabrication. Feng and Chen [13] demonstrated the use of micromachining technology to create arbitrary-shaped IPMC transducers to accurately shape micro-feature-sized devices without knife cutting or laser 
burning. In addition, Feng and Tsai successfully fabricated column-structured three-dimensional four-electrode IPMC actuators with an optical fiber enclosed to conduct laser light for surgical applications [14]. Recently, the unique IPMC tweezers made by using the microfabrication technique of arbitrary shaping and three-dimensional multi-electrode motion control concept have been demonstrated [15]. The tweezers consist of two main parts: a motion element and a clamping head. With novel multiple electrodes design, the clamping head can move multi-directionally, and it can also provide an adjustable clasping force through electrical voltage control.

Research has also focused on fabricating electrodes of IPMCs with versatile materials. Platinum is the most common material used for electrodes because of its stability and excellent conductivity. Park and Kim [16], however, recently reported a multi-field responsive IPMC using nickel as the electrode material due to its ability to respond to multiple driving forces, such as electric and magnetic. Another benefit of using nickel is replacing precious metals (e.g., platinum or gold) to reduce the cost of the fabrication process.

This paper presents our successful attempt to develop a three-dimensional multi-electrode IPMC clamp with tuning fork-shaped construction. Nickel was applied as the electrode material to achieve the most cost-effective device. The detailed design, fabrication, and testing results are described in the following section.

\section{Device Design and Working Principle}

Figure 1 shows the prototype design of the tuning fork-shaped IPMC clamp. The entire length of the device is $15 \mathrm{~mm}$ with a uniform thickness of $2 \mathrm{~mm}$. It is composed of two portions: a U-type active clamp and a column handle. The clamp and column handle portion

Guo-Hua Feng received the M.S. degrees in mechanical engineering from National Central University, Taiwan, and University of California, Los Angeles, USA, in 1995 and 2000, respectively and Ph.D. degree in electrical engineering from University of Southern California, Los Angeles, USA, in 2004. In spring 2005, he joined the National Chung Cheng University, Taiwan, and is currently an associate professor in the Department of Mechanical Engineering. He worked as a graduate research assistant with the Department of Electrical Engineering at University of Southern California, Los Angeles, in the MEMS Group from 2000 to 2004. From 1998 to 2000, he also worked as a graduate research assistant on micronozzle project in the Department of Mechanical Engineering and Aerospace Engineering at the University of California, Los Angeles. Before coming to the United States, he had been a lecturer in the Department of Mechanical Engineering at Chin-Ming College, Taiwan. His research interests include microfluidic devices, microfabrication processing technology, acoustic transducers, and bio-MEMS devices.

Wei-Lun Huang received the B.S. degree in Electronic Engineering from National Kaohsiung Normal University, Taiwan, in 2010. He is currently a graduate student in National Chung Cheng University and his research focus is in the area of bio-MEMS related device fabrication. were each designed to be half the length of the device. The active clamp was designed with longer fingers and with larger width and thickness than previously reported transducers [15]. The purpose of this design was to enable greater force output of the U-type clamp through larger displacement. The handle part plays the role of structurally merging the two fingers of the clamp, and is also responsible for the electrical signal transmission from the actuating power source to the fingers.

Three active electrodes were fabricated in this novel clamp transducer. One electrode was plated on the inner surface of the clamp portion; the other two electrodes were plated onto the individual outer surface of the clamp portion. Three narrow-plated metal electrodes plated on the handle part of the device functioned as the wire to connect active electrodes to the power source (Figure 1). To operate the tuning fork-shaped clamp, the two outer surface electrodes were connected, and the driving signals were sent to the outer and inner surface electrodes of the device.

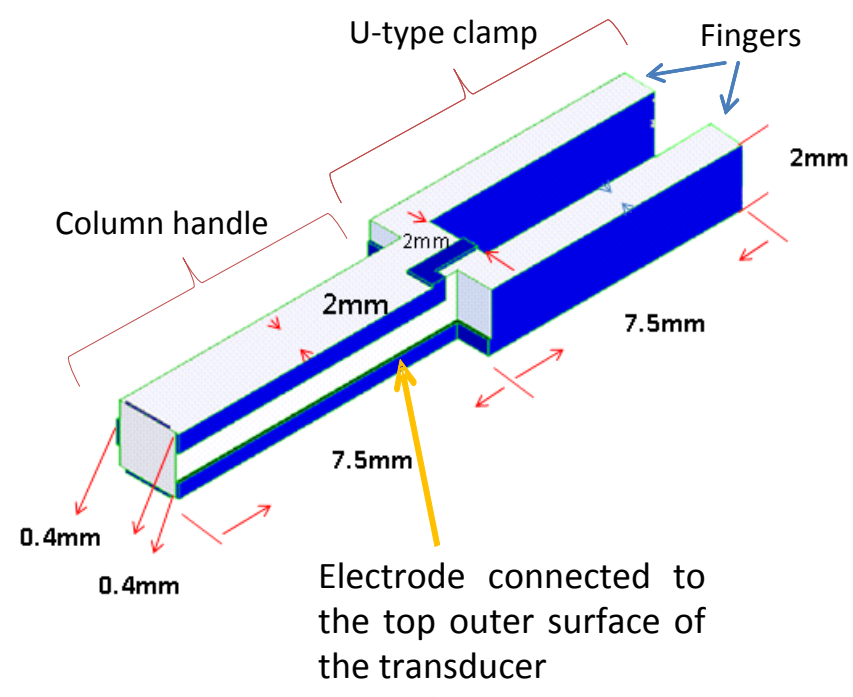

(a)

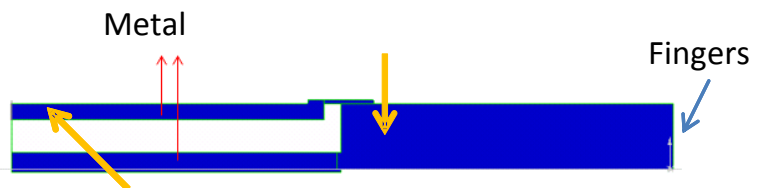

Electrode connected to the inner surface of the transducer

(b)

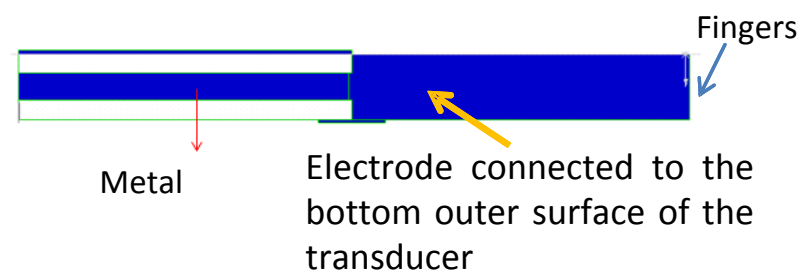

(c)

Figure 1. Schematic diagram of the tuning fork-shaped IPMC clamp. 


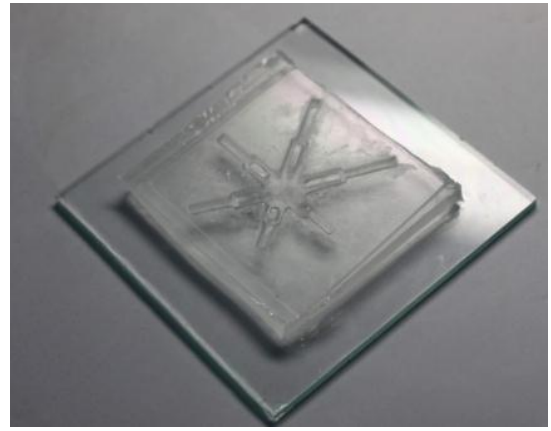

Figure 2. Fabricated PDMS mold for processing tuning fork-shaped Nafion devices.

\section{Device Fabrication}

A $1 \mathrm{~mm}$ deep cavity was constructed on a flat plane by gluing glass slides around a $10 \mathrm{~cm} \times 10 \mathrm{~cm}$ glass plate, making the walls of the $1 \mathrm{~mm}$ cavity. This was followed by pipetting SU8-100 photoresist to fill the cavity. A $1 \mu \mathrm{m}$-thick parylene membrane was conformally coated on the glass cavity to prevent SU8-formed templates from sticking on the glass mold during subsequent removal. After soft baking, SU8 was patterned as the designed templates, including a device region for creating the tuning fork-shaped IPMC clamps and a refill region to replenish the liquid Nafion solution. Following this, two identical SU8 templates peeled from the glass cavity were aligned, and instant glue was applied to join the templates to complete a 2 - $\mu$ m-thick template with multiple distinct tuning fork-shaped IPMC devices. Next, the $2 \mu \mathrm{m}$-thick SU8 template was glued to the center region of another $10 \mathrm{~cm} \times 10 \mathrm{~cm}$ glass plate, and 4-mm-thick glass slides were glued around the glass plate to form a cavity with the SU8 template inside. Liquid PDMS was poured to fill the cavity, while being careful to avoid the formation of air bubbles at the liquid PDMS/SU8 template interface. The shaped PDMS mold was solidified at room temperature after $12 \mathrm{~h}$, and was subsequently removed from the glass cavity.

The PDMS mold was glued to a $10 \mathrm{~cm} \times 10 \mathrm{~cm}$ glass plate for subsequent Nafion device fabrication (Figure2). A thin parylene was deposited onto the entire unit, done so for the following two purposes: 1) reducing the surface adhesion force between the liquid Nafion solution and the PDMS mold to enable the device to maintain a planar upper surface [12]; and 2) easy peeling of the solidified Nafion devices from the PDMS mold. Liquid Nafion was then added into the refill region to fill the whole mold. The poured Nafion solution solidified after approximately one and a half hours. The shaped Nafion element was removed, and the refill region was sliced to obtain Nafion devices of various designs.

Figure 3 illustrates the fabrication process to form multiple electrodes on the device. A piece of patterned

tape was attached to the Nafion device as the shadow mask to define the inactive regions of the transducer. The tape was patterned using a laser-machining tool controlled by a computer. AZ5214 photoresist was then sprayed onto the tape-attached Nafion device to form the selective protection layer. The shadow mask was subsequently removed, and chemical electroding processing was executed to plate nickel electrodes on the active regions of the clamp transducer.

The nickel plating procedure is as follows: The concentration of $1 \mathrm{M}$ nickel sulfate solution was first prepared by adding $39.42 \mathrm{~g}$ nickel sulphate pellets into $150 \mathrm{~g}$ deionized water at room temperature. The processed Nafion devices were immersed in the solution for $12 \mathrm{~h}$, and then washed with deionized water for further reduction reaction. An amount of $20 \mathrm{mg} \mathrm{\textrm {LiBH } _ { 4 }}$ powder was added into $30 \mathrm{~g}$ deionized water. After proper agitation to form a homogeneous solution, the devices were immersed in the solution. The reaction was performed for $2 \mathrm{~h}$, followed by another $2 \mathrm{~h}$ reduction process through replacing a new $\mathrm{LiBH}_{4}$ reduction solution. As a final step, the processed transducers were treated with sodium hydroxide solution for $12 \mathrm{~h}$ to perform both ion-exchange processing and patterned photoresist removal. After this step, the tuning fork-shaped IPMC clamp was completed and ready for testing. Figure 4 shows the fabrication result of the IPMC clamp.

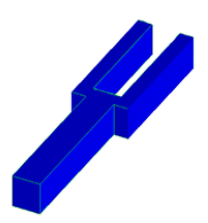

1. Attach patterned tape onto the solidified Nafion device.

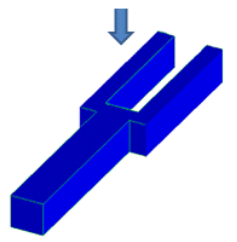

2. Apply photoresist to the selective area of the device.

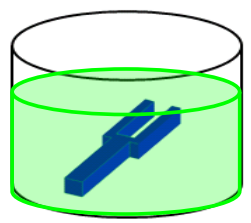

3. Immerse the device into Nickel Sulfate solution for 12 hours.

Figure 3. Fabrication process of nickel-electrode IPMC clamp transducers. 


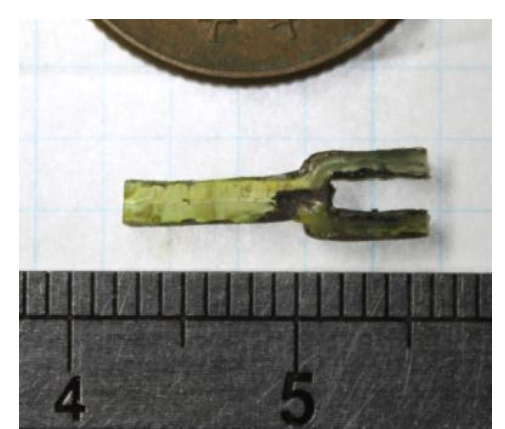

(a)

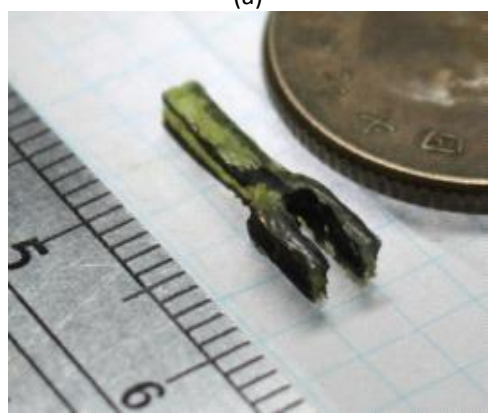

(b)

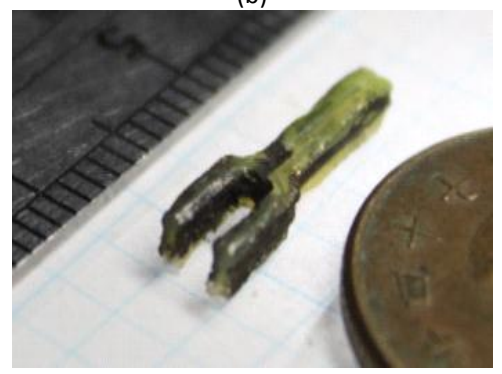

(c)

Figure 4. Photographs of the fabricated IPMC clamp from different viewpoints.

\section{Experimental Methods}

Two experiments were designed to evaluate the performance of the developed IPMC clamps: 1) actuating the IPMC clamp without interactions with external objects, that is, only driving the transducer to reach an open or closed state; and 2) actuating the transducer to clamp an external object, with the clamped object being intentionally removed by a sudden external force. This was conducted to simulate the biomimetic behavior of humans of using the thumb and forefinger to pinch an object, and when an external applied force grabs that object away suddenly. The electrical property and dynamic motion of the IPMC clamp were recorded during the experiments.

To operate the IPMC clamp transducer, a tool with three electrode pads was used to anchor the developed IPMC clamp and transmit control signals (Figure 5). The tool was commercially available metal tweezers that maintained a closed state with no external force applied. Three electrical pads made with trimmed conductive copper tape were aligned to the electrode locations of the IPMC clamp and glued to the front end of the tweezers. To prevent a short circuit, scotch tape was applied to the copper tape/tweezers interface as an insulation layer. Wires were individually soldered to the three copper tape electrodes to connect driving signals from a function generator. The electric conductivity was assessed before testing to ensure that the control signal could be transmitted to the device from the function generator.

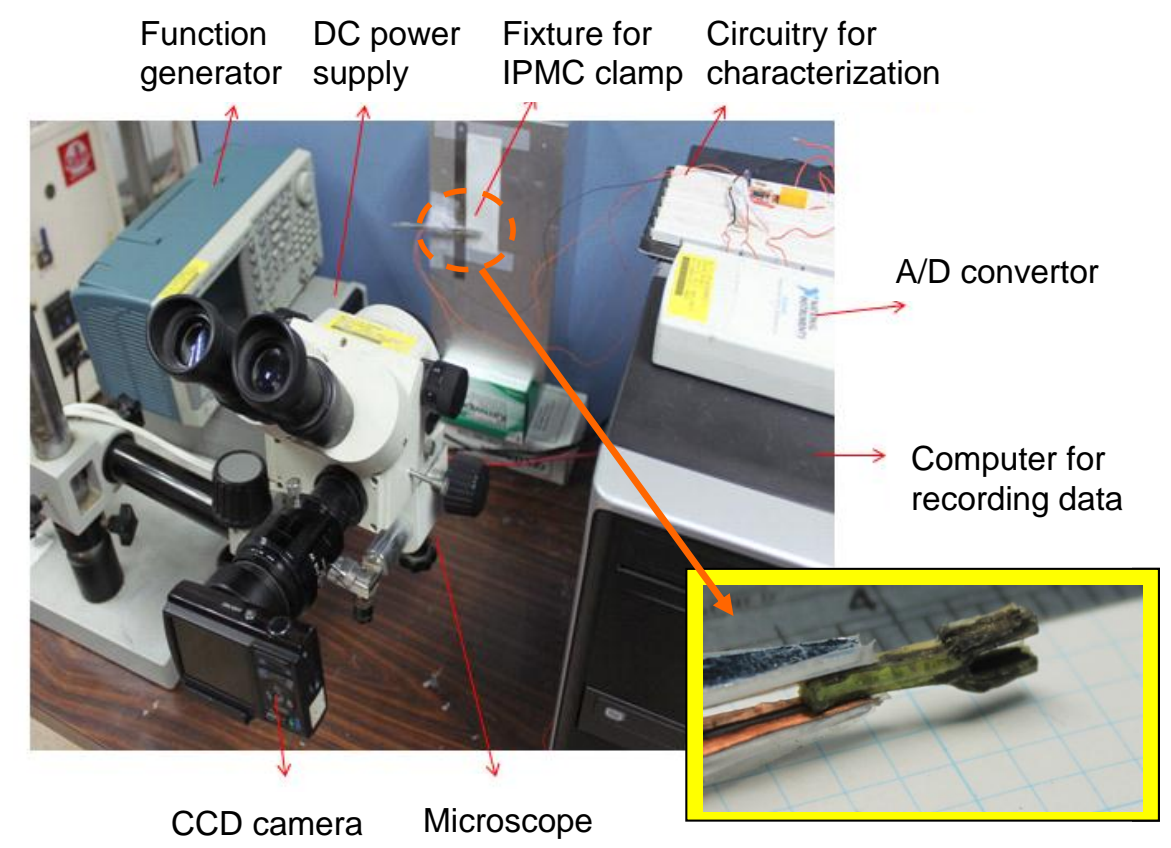

Figure 5. Experimental setup for characterizing the performance of the fabricated IPMC transducers. 
A simple and useful circuitry, as described in [2], was set up to drive the device and acquire the current consumption and voltage drop across the IPMC transducer. An appropriate resistor of $3.3 \Omega$ was selected to connect in series with the IPMC clamp in this case. The proper resistance value allows the voltage drops across the selected resistor and IPMC device to be of the same degree, meaning the voltage drop across the resistor is not too small for acquisition.

The dynamic motion of the IPMC clamp was characterized using the setup of a microscope with a high-resolution CCD video camera [9]. The camera captured images of the motion of the side profile of the fabricated IPMC clamp (i.e., in the direction perpendicular to the electrodes) with a frame rate of 10 fps. The captured images could be further analyzed using commercial software to delineate the displacement of a specified point on the transducer with a displacement resolution of $10 \mu \mathrm{m}$.

In the first experiment, we used $0.01 \mathrm{~Hz}$ square waves with amplitudes of $4 \mathrm{~V}$ and $8 \mathrm{~V}$ (zero voltage bias) supplied by a function generator as the driving signals. Figure 6 indicates three specific points that characterized the displacement in the first experiment. Points $A$ and $B$ are located at the frontend middle points of the upper and lower fingers. Point $C$ is positioned at the middle point of the handle part, which is $2 \mathrm{~mm}$ away from the inner edge of the U-type clamp. An actuating DC voltage of $4 \mathrm{~V}$ provided by a power supply was used in the second experiment. The external object used in the second experiment was a soft small column made of PDMS with the dimensions of $1.2 \mathrm{~mm} \times 1 \mathrm{~mm} \times 1 \mathrm{~mm}$. The small PDMS column was horizontally placed close to the front end of the lower finger before the actuating signal was applied to the IPMC clamp. In both experiments, a 3.5-mm-long backend portion of the handle of the transducer was anchored to the fixture.

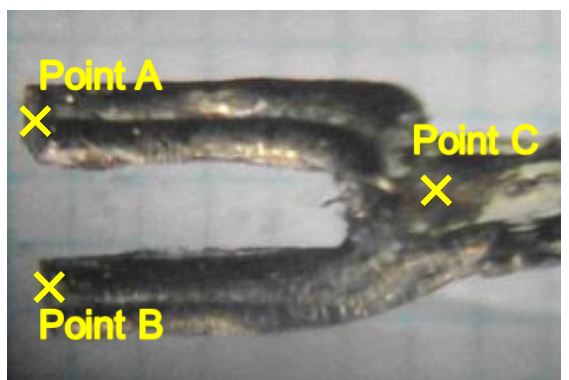

Figure 6. Illustration of three specific points to measure the displacements of the IPMC clamp.

\section{Results and Discussion}

Figure 7 shows the measured voltages across and currents flowing through the transducer when $4 \mathrm{~V}$ and 8 $\mathrm{V}$ driving signals were provided. During actuation of the IPMC clamp, the polarity switching and the polarity of the $0.01 \mathrm{~Hz}$ square wave were unspecified when turning on the function generator. Hence, the driving voltages across the transducer exhibited a positive value and a negative value during application of external voltages of $4 \mathrm{~V}$ and $8 \mathrm{~V}$, respectively. In addition, the first switching points occurred at approximately $35 \mathrm{~s}$ and $20 \mathrm{~s}$, and switched the polarities every alternate $50 \mathrm{~s}$ for the $4 \mathrm{~V}$ and $8 \mathrm{~V}$ cases. The consuming current of the transducer and voltage across the transducer were proportional to the external applied voltages when reaching a steady state.

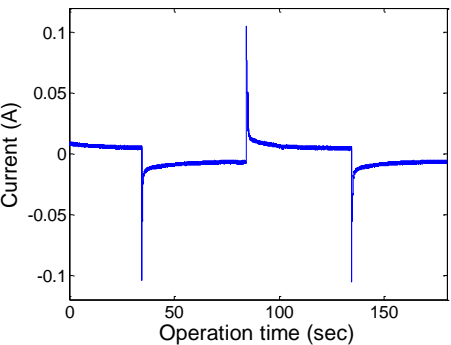

(b)

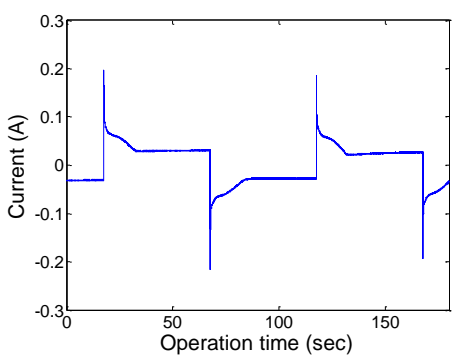

(d)

Figure 7. (a) and (c): voltages across the IPMC transducer for external applied voltages of $4 \mathrm{~V}$ and $8 \mathrm{~V}$, respectively. (b) and (d): the corresponding currents flowing through the IPMC transducer. 


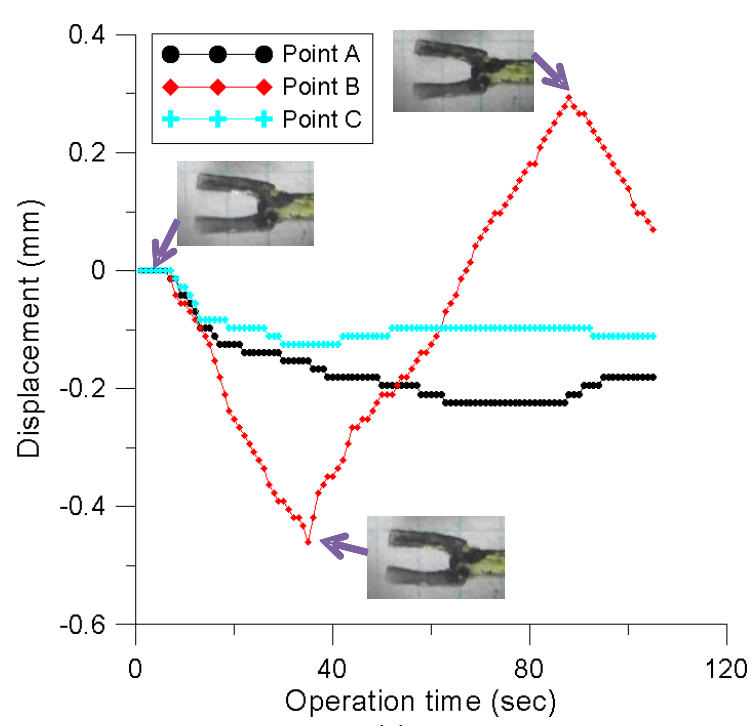

(a)

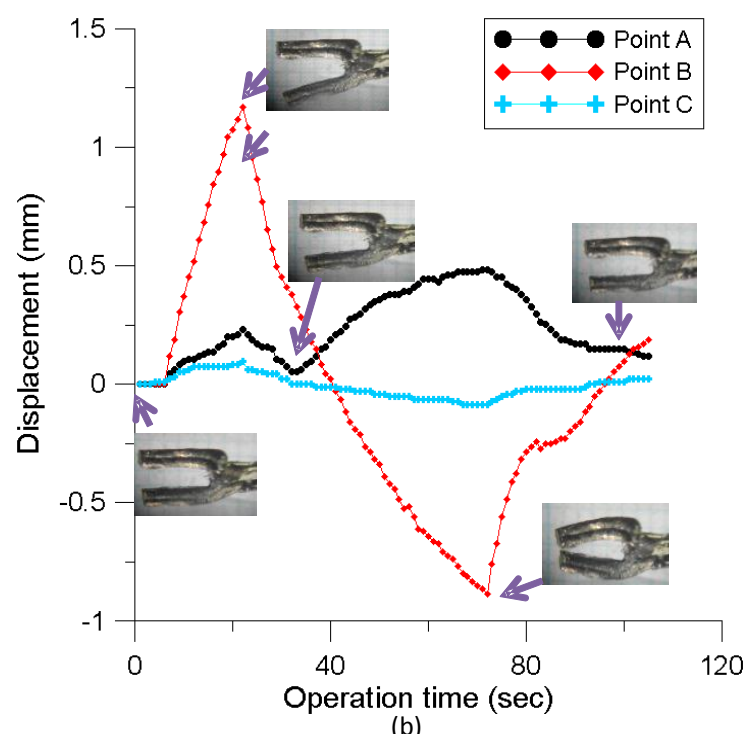

(b)

Figure 8. Measured displacements (positive downward) at three specific points (ref. Figure 6) based on the driving conditions shown in Figure 7.

Figure 8 shows the measured displacement results of points $A, B$, and $C$, which correspond to the driving signals shown in Figure 7. The coordinate is defined as positive down in this study. We observed the motion at point B exactly as expected, moving upward (i.e., toward a fully closed clamp state) when positive voltage was applied to the outer electrodes of the clamp and moving downward when negative voltage was applied to the outer electrodes of clamp. This was caused by the hydrocations moving toward the cathode of the IPMC transducer [8]. Point A shows the motion as expected in the interval between $35 \mathrm{~s}$ and $85 \mathrm{~s}$ and between $32 \mathrm{~s}$ and $72 \mathrm{~s}$ for the $4 \mathrm{~V}$ and $8 \mathrm{~V}$ cases, respectively. The two time intervals were within the second-half period of the driving square wave. For the $8 \mathrm{~V}$ case, the second-half period of actuating signal (22 s-72 s) was supposed to cause the clamp to approach a fully closed state.
However, a downward displacement was displayed in 22 s-32 s. Moreover, the displacement of point A exhibited a relatively small value compared to point $B$.

Valuable information was obtained after assessing the displacement at point $C$. The unsymmetrical design caused the displacement at point $C$ to vary as a function of time. The same displacement trend was observed in points $B$ and $C$ before the first polarity switching (for both $4 \mathrm{~V}$ and $8 \mathrm{~V}$ ) and obviously for the larger actuation voltage case after switching. Results indicate the crucial role of the connected electrodes traveling through the side surfaces of the transducer and their possessing of various layouts. The middle connected electrode design on the lower handle part of the transducer induced a larger motion effect than the two-side electrode design on the upper handle part, especially at the neck region of the transducer (close to the end portion of two fingers). This may be a valuable topic for further exploration. However, because this paper focuses on the development of the novel IPMC clamp, this discussion will be presented in the following papers.
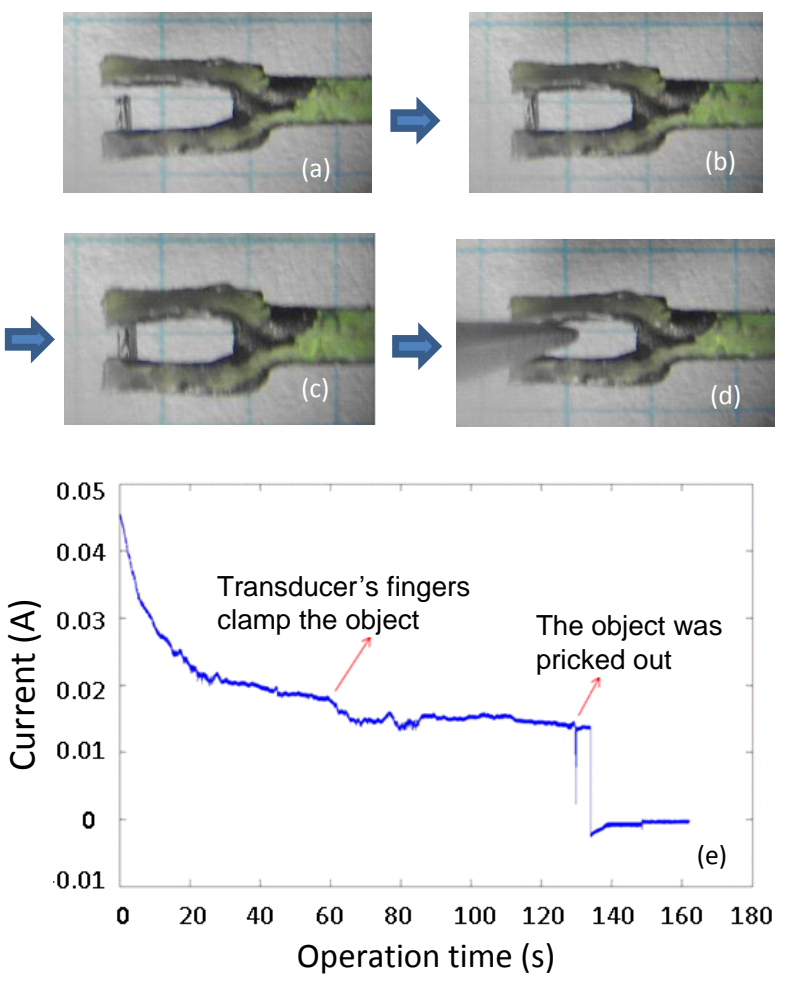

Figure 9. Photographs of the IPMC transducer interacting with an object: (a) Initial state; (b) Clamping an object; (c) Squeezing the object; (d) The object being pulled out by a sudden force; (e) The corresponding feedback current response in the process (a)-(d).

Figure 9 demonstrates that the developed IPMC clamp successfully clamped a soft object. Initially, the object was placed on the lower finger of the clamp. The fingers clamped the object at $60 \mathrm{~s}$ after a DC $4 \mathrm{~V}$ was applied (Figure 9(b)). Subsequently, the clamp continuously supplied force to squeeze the object. The 
object was intentionally pulled out at approximately $128 \mathrm{~s}$ (Figure 9(d)). This test demonstrated that the squeezing behavior of the IPMC clamp lasted for more than $1 \mathrm{~min}$. In addition, our earlier work [13] suggested that the current signal could be an excellent sensing signal for identifying the behavior of IPMC transducers. This was verified by assessing the current flowing through the transducer (Figure 9(e)). We monitored the moment of the object being clamped and pulled out using the feedback current signal.

\section{Conclusion}

A novel tuning fork-shaped IPMC clamp was designed, fabricated, and tested. The clamp transducer was successfully developed using a cost-effective nickel-plating technique along with a micromachining process. According to experimental results, clamping on/off motions were observed, and the maximum displacement between the two fingers of the clamp reached $2 \mathrm{~mm}$ at a square wave of amplitude $8 \mathrm{~V}$ actuation. The two fingers of the clamp exhibited different dynamic behaviors due to unsymmetrical design, which requires further investigation. The developed IPMC clamp also demonstrated the ability to pinch an object for more than $1 \mathrm{~min}$. This may be useful in soft robotics and other applications.

\section{Acknowledgement}

The authors would like to thank National Science Council in Taiwan for financial support.

\section{References}

[1] S. K. De, N. R. Aluru, B. Johnson, W. C. Crone, D. J. Beebe, and J. Moore, "Equilibrium swelling and kinetics of ph-responsive hydrogels: Models, experiments, and simulations," Journal of Microelectromechanical Systems, vol. 11, no. 5, pp. 544-555, 2002 doi: 10.1109/JMEMS.2002.803281

[2] G. H. Feng and J. W. Tsai, "Investigation of electrical to mechanical energy conversion of a three-dimensional four-electrode multidirectional-controllable ipmc transducer with/without an optical fiber enclosed," Smart Materials and Structures, vol. 20, no. 1, p. 015027, 2011.

doi: $10.1088 / 0964-1726 / 20 / 1 / 015027$
[3] K. J. Kim and M. Shahinpoor, "Ionic polymer metal composites: li. Manufacturing techniques," Smart Materials and Structures, vol. 12, no. 1, pp. 65-79, 2003.

doi: $10.1088 / 0964-1726 / 12 / 1 / 308$

[4] W. J. Yoon, P. G. Reinhall, and E. J. Seibel, "Analysis of electro-active polymer bending: A component in a low cost ultrathin scanning endoscope," Sensors and Actuators A: Physical, vol. 133, no. 2, pp. 506-517, 2007. doi: 10.1016/j.sna.2006.04.037

[5] I. S. Rajay Vedaraj, S. Parijaat, and B. V. A. Rao, "Material analysis for artificial muscle and touch sensing of cooperative biomimetic manipulators," The International Journal of Advanced Manufacturing Technology, 2011. doi: $10.1007 / \mathrm{s} 00170-011-3640-8$

[6] I. S. Park, S. M. Kim, D. Pugal, L. Huang, S. W. Tam-Chang, and K. J. Kim, "Visualization of the cation migration in ionic polymer-metal composite under an electric field," Applied Physics Letters, vol. 96, no. 4, p. 043301, 2010. doi: $10.1063 / 1.3293290$

[7] F. Carpi, R. Kornbluh, P. Sommer-Larsen, and G. Alici, "Electroactive polymer actuators as artificial muscles: Are they ready for bioinspired applications?," Bioinspiration \& Biomimetics, vol. 6, no. 4, pp. 045006-045006, 2011

[8] B. J. Akle and D. J. Leo, "Characterization and modeling of extensional and bending actuation in ionomeric polymer transducers," Smart Materials and Structures, vol. 16, no. 4, pp. 1348-1360, 2007. doi: $\underline{10.1088 / 0964-1726 / 16 / 4 / 049}$

[9] S. Liu, R. Montazami, Y. Liu, V. Jain, M. Lin, X. Zhou, J. R. Heflin, and Q. M. Zhang, "Influence of the conductor network composites on the electromechanical performance of ionic polymer conductor network composite actuators," Sensors and Actuators A: Physical, vol. 157, no. 2, pp. 267-275, 2010. doi: $10.1016 /$ j.sna.2009.11.022

[10] G. H. Feng, "Numerical study on dynamic characteristics of micromachined ionic polymer metal composite devices based on molecular-scale modeling," Computational Materials Science, vol. 50, no. 1, pp. 158-166, 2010. doi: $10.1016 /$ j.commatsci.2010.07.020

[11] G. H. Feng and R. H. Chen, "Fabrication and characterization of arbitrary shaped $\mu \mathrm{IPMC}$ transducers for accurately controlled biomedical applications," Sensors and Actuators A: Physical, vol. 143, no. 1, pp. 34-40, 2008. doi: $10.1016 /$ j.sna.2007.06.032

[12] M. Shahinpoor, Y. Bar-Cohen, J. O. Simpson, and J. Smith, "Ionic polymer-metal composites (IPMCs) as biomimetic sensors, actuators and artificial muscles - a review," Smart Materials and Structures, vol. 7, no. 6, pp. R15-R30, 1998. doi: 10.1088/0964-1726/7/6/001 
[13] G. H. Feng and R. H. Chen, "Improved cost-effective fabrication of arbitrarily shaped $\mu$ ipmc transducers," Journal of Micromechanics and Microengineering, vol. 18, no. 1, p. 015016, 2008. doi: $10.1088 / 0960-1317 / 18 / 1 / 015016$

[14] G. H. Feng and J. W. Tsai, "Micromachined optical fiber enclosed 4-electrode ipmc actuator with multidirectional control ability for biomedical application," Biomedical Microdevices, vol. 13, no. 1, pp. 169-177, 2010. doi: $10.1007 / \mathrm{s} 10544-010-9482-6$
[15] G. H. Feng and J. W. Tsai, "3D omnidiectional contollable elastic ipmc tweezer with self-sensing and adjustable clamping force abilities for biomedical applications," in 16th International Solid-State Sensors, Actuators and Microsystems Conference (TRANSDUCERS), Beijing, China, 2011, pp. 1725-1728.

doi: 10.1109/TRANSDUCERS.2011.5969609

[16] I. S. Park and K. J. Kim, "Multi-fields responsive ionic polymer-metal composite," Sensors and Actuators A: Physical, vol. 135, no. 1, pp. 220-228, 2007. doi: 10.1016/i.sna.2006.07.014 